\title{
Big Data Analytics for Mass Casualty Incident (MCl) Situational Awareness
}

\author{
Catherine Ordun*1, Timothy Davis ${ }^{5}$, Brante Goode ${ }^{2}$, Dean Ross ${ }^{3}$ and Mike Hopmeier \\ ${ }^{1}$ Strategic Innovation Group, Booz Allen Hamilton, Atlanta, GA, USA; ${ }^{2}$ Office of Public Health Preparedness and Response, Centers \\ for Disease Control and Prevention, Atlanta, GA, USA; ${ }^{3}$ National Park Service, Washington, DC, USA; ${ }^{4}$ Unconventional Concepts, \\ Inc., Reston, VA, USA; ${ }^{5}$ National Disaster Medical System, Assistant Secretary of Preparedness and Response, Washington, DC, \\ USA
}

\section{Objective}

Discuss how different big-data analytics, techniques, and tools including open source platforms, cloud analytics, social media, crowdsourcing, and geospatial visualization can be used to quickly achieve situational awareness within seconds of a MCI, for use by pre-hospital responders, healthcare workers, and policy makers.

\section{Introduction}

A variety of big data analytics, techniques and tools including social media analytics, open source visualizations, statistical anomaly detection, use of Application Programming Interfaces (APIs), and geospatial mapping, are used for infectious disease biosurveillance. Using these methodologies, policy makers and practitioners detect and monitor outbreaks across the world near real time, in multiple languages, 24/7. The non-infectious disease community, namely critical care, injury, and trauma stakeholders, currently lack this level of sophistication. To respond to most MCIs like a terrorist bombing, validated, real-time information is typically available via closed radio channels and limited to a specific set of emergency responders. Health care workers, policy makers, and citizens reach for news, radio, and Internet sources to characterize casualties and hazards, and increasingly social media. During the Boston Marathon bombing, witnesses began posting tweets seconds after the bombing and 15 seconds before $\mathrm{CNN}$ reported the incident. Current trauma data sets are unhelpful for real time response, including trauma registries that are used for hospital performance after an incident, and disaster databases consist of secondary reporting used for academic research purposes.

\section{Description}

The purpose of this panel is to discuss how different analytic tools can be used to achieve more rapid and insightful situational awareness during a MCI. It will discuss how innovative analytics that are used in biosurveillance such as outbreak detection and monitoring,, could potentially be applied to MCIs. The panel will share case studies of their respective agency's challenges in gathering information during a MCI, and highlight some potential ways that data and analytics could be used to improve situational awareness about casualty volume, injuries and trauma, secondary hazards, and population movement during the first moments of a MCI. The panel is seeking to solicit feedback about which data, tools, and methods might be most applicable to the syndromic surveillance community in creating a MCI analytics tool.

\section{Audience Engagement}

Moderator will begin by polling audience on a) their work with MCIs, b) use of analytics in their daily tasks, c) what challenges they have faced dealing with "information blackouts" in MCIs. Each panelist will discuss a brief example from their agency or organization in dealing with a MCI from different points of view: a) medical operations (ASPR, DoD, DHS), b) epidemiology and public health ( $\mathrm{CDC} /$ Vermont), and $\mathrm{c})$ the mass gathering and population monitoring (NPS).

\section{Keywords}

mass casualty; analytics; open source; informatics; trauma

\section{Acknowledgments}

- Ms. Catherine Ordun (Moderator), MBA, MPH, Associate, Booz Allen

Hamilton, Strategic Innovations Group, Atlanta, GA

- Dr. Tim Davis (Panelist), MD, MPH, Assistant Secretary of Preparedness and Response, Chief Medical Officer for National Disaster Medical System (NDMS), Washington, DC

- Mr. Brant Goode (Panelist), RN/BSN, MPH, Captain, U.S. Public Health Service, CDC Career Epidemiology Field Officer assigned to Vermont Dept. of Health

- Mr. Dean Ross (Panelist), Deputy Chief, Law Enforcement, Security, and Emergency Services, Branch Chief of Emergency Services at National Park Service Office of Public Health, National Park Service

- Mr. Michael Hopmeier (Panelist), MS, President, Unconventional Concepts, Inc., Reston, VA

- Mr. KC Decker (Co-Author), ASEM, Senior Associate, Booz Allen Hamilton, Strategic Innovations Group, Atlanta, GA

\section{References}

http://www.ncbi.nlm.nih.gov/pmc/articles/PMC3706072/: Cassa CA, Chunara R, Mandl K, Brownstein JS. Twitter as a Sentinel in Emergency Situations: Lessons from the Boston Marathon Explosions. PLOS Currents Disasters. 2013 Jul 2. Edition 1. doi: 10.1371/currents. dis.ad70cd1c8bc585e9470046cde334ee4b.

\section{${ }^{*}$ Catherine Ordun}

E-mail: ordun_catherine@bah.com 